\title{
Relación entre la presencia del portero y las variables pedagógicas que definen las tareas en el fútbol. Un estudio de caso
}

\author{
Gamonales, José M.; León, Kiko; Muñoz-Jiménez, Jesús \\ Relación entre la presencia del portero y las variables pedagógicas que definen las tareas en el fútbol. Un \\ estudio de caso \\ MHSalud, vol. 18, núm. 1, 2021 \\ Universidad Nacional, Costa Rica \\ Disponible en: http://www.redalyc.org/articulo.oa?id=237064193004 \\ DOI: https://doi.org/10.15359/mhs.18-1.4
}

Esta obra está bajo una Licencia Creative Commons Atribución-NoComercial-SinDerivar 3.0 Internacional 


\title{
Relación entre la presencia del portero y las variables pedagógicas que definen las
} tareas en el fútbol. Un estudio de caso

\author{
Relationship Between the Presence of the Goalkeeper and the Pedagogic Variables that Define the Football Tasks. A \\ Case Study \\ Relação entre a presença do goleiro e as variáveis pedagógicas que definem as tarefas no futebol. Um estudo de caso
}

José M. Gamonales

Universidad de Extremadura, España

martingamonales@unex.es

(iD http://orcid.org/0000-0002-2444-1535

Kiko León

Universidad de Extremadura, España

fleon@unex.es

(D) http://orcid.org/0000-0002-3333-482X

Jesús Muñoz-Jiménez

Universidad de Extremadura, España

Universidad Autónoma de Chile, Chile

suliwan@unex.es

jesus.munoz@uautonoma.cl

(D) http://orcid.org/0000-0003-1283-5227
DOI: https://doi.org/10.15359/mhs.18-1.4

Redalyc: http://www.redalyc.org/articulo.oa? $\mathrm{id}=237064193004$
Recepción: 20 Mayo 2020

Aprobación: 20 Agosto 2020

\section{Resumen:}

La presente investigación tiene el objetivo de analizar las variables pedagógicas que se incluyen en las tareas de entrenamiento diseñadas por el entrenador de fútbol-base, categoría Benjamín (edad comprendida entre 9 y 10 años), respecto a la presencia o no del portero. Con tal fin, se analizaron un total de 46 tareas de entrenamiento desarrolladas durante 2 meses de la etapa formativa. Se utilizó la herramienta SIATE para la categorización y análisis de las siguientes variables pedagógicas de dichas tareas: Presencia deportero (POR), Situación de Juego (SJ), Fase de Juego (FJ), Tipo de Contenido (CONT-G), Medio de Iniciación Deportiva (MIE), Nivel de Oposición (NO), y Linea de Juego (LJ). Los resultados muestran que el entrenador diseña tareas de entrenamiento de $8 \mathrm{x}$ 8 , mixta . competitivas con presencia de portero, y tareas de entrenamiento $1 \mathrm{x} 0$, así como ejercicio de aplicación compleja para el calentamiento sin presencia de portero. Se concluye que existen diferencias significativas entre la variable presencia de portero y el modo de actuar del entrenador.

PALABRAS ClaVE: fútbol, formación, entrenamiento, portero.

\section{Abstract:}

This research aims to analyze the pedagogical variables included in the training tasks designed by the soccer-base coach, Benjamin category (age between 9 and 10 years), regarding the presence or not of the goalkeeper. To do this, a total of 46 training tasks framed during 2 months of the training stage were analyzed. For the categorization of tasks, the SIATE tool was used, to analyze the pedagogical variables: Presence of Goalkeeper (PG), Game Situation (GS), Game Phase (GH), Content Type (CONT-T), Sports Initiation Medium (SIM), Opposition Level (OL), and Line of Play (LP). The results show that the coach designs $8 \times 8$, mixed and competitive training tasks with the presence of a goalkeeper, and 1 x 0 training tasks, complex application exercise for warm-up without the presence of a goalkeeper. Also, there are significant differences between the variable presence of goalkeeper and the coach's way of acting.

KEYWORDS: football, formation, training, goalkeeper.

\section{Resumo:}

A presente pesquisa tem como objetivo analisar as variáveis pedagógicas que integram as tarefas de treinamento elaboradas pelo técnico de futebol juvenil da categoria Benjamin (idade entre 9 e 10 anos), no que se refere à presença ou não do goleiro. Para 
MHSALUd, ISSN: 1659-097X, 18(1), ENERo-Junio, 2021, pP 1-14

GAMONALES, LEÓN Y MuÑoz-JimÉnEZ

isso, foram analisadas 46 tarefas de treinamento desenvolvidas durante 2 meses da etapa de treinamento. A ferramenta SIATE foi utilizada para a categorização e análise das seguintes variáveis pedagógicas dessas tarefas: Presença do Goleiro (PG), Situação do Jogo (SJ), Fase do Jogo (FJ), Tipo de Conteúdo (T-CONT), Meio Esporte de Iniciação (MEI), Nivel de Oposição (NO), e Linha de Jogo (LJ). Os resultados mostram que o técnico projeta tarefas de treinamento $8 \times 8$, mistas e competitivas com a presença de goleiro, e tarefas de treinamento 1 x 0, além de um complexo exercício de aplicação para aquecimento sem a presença de goleiro. Conclui-se que existem diferenças significativas entre a variável presença do goleiro e a forma de atuação do técnico.

Palavras-Chave: futebol, formação, treinamento, goleiro.

\section{INTRODUCCIÓN}

El portero de fútbol tiene unas características diferenciadas respecto a cualquier otro jugador de campo, como consecuencia del rol que desempeña durante el juego, el cual contiene un elevado grado de responsabilidad (Bazmara et al., 2013). En los últimos años, ha experimentado una evolución en el nivel técnico-táctico condicionado por las modificaciones reglamentarias, sumada al interés de los entrenadores para que el guardameta actué como última línea de defensa y primer atacante en la construcción del juego ofensivo (Shafizadeh et al., 2015).

En la literatura científica, los estudios técnico-científicos sobre el portero de fútbol son escasos y los que existen analizan aspectos muy concretos, como las lesiones deportivas (Hägglund et al., 2013; Pidemunt et al., 2012; Putukian, 2004), sus factores psicológicos ante lanzamientos de penalti (Furley et al., 2016; Lidor et al., 2012; Navarro et al., 2013), la eficacia de su comportamiento técnico-táctico (Lapresa et al., 2018), perfiles fisiológicos (Di Salvo et al., 2008; Montesano, 2016) o sus factores perceptivos y de control motor (Dicks et al., 2011; Gegenfurtner et al., 2011). Además, se encuentran trabajos relativos al análisis del juego del portero de élite (Oberstone, 2010; Sainz de Baranda et al., 2008), pero es escasa la producción científica cuyo objeto de estudio sea el portero de fútbol-base (Lapresa et al., 2018; Sainz de Baranda et al., 2005) y aun menores, las investigaciones relacionadas con el diseño de las tareas de entrenamiento (Ortega-Toro y Sainz de Baranda, 2003).

El entrenamiento en fútbol-base tiene un importante papel en la adquisición y el perfeccionamiento de las habilidades técnico-tácticas (Silva et al., 2005), así como de los aspectos tecnomotrices. Las habilidades neuromusculares son la base para el posterior aprendizaje y desenvolvimiento de las acciones técnicotácticas. Las tareas que diseñan los entrenadores son las unidades más concretas del proceso de planificación y programación del entrenamiento (Sáenz-López et al., 2006), que permiten desarrollar los objetivos planteados a través de la práctica de contenidos deportivos. Por ello, es necesario implementar, de forma implícita y explícita, diferentes concepciones metodológicas durante los entrenamientos (Ibáñez et al., 2016). La selección de una metodología repercute directamente tanto en el diseño como en la selección de las tareas de entrenamiento y, por consiguiente, en el proceso formativo del deportista (Mancha-Triguero et al, 2018).

Durante el entrenamiento, los encargados de este manipulan diferentes variables para el diseño de las tareas (Gracia et al., 2014), independientemente del posicionamiento metodológico en la intervención práctica (Gamonales et al., 2020; Ibáñez et al., 2016), con la finalidad de desarrollar actividades óptimas y adaptadas a los jugadores que configuran los equipos. Al igual que otras modalidades deportivas, el fútbol no es una ciencia, pero esta puede ayudar a mejorar el rendimiento (Stølen et al., 2005), mostrando los aspectos relevantes que se desarrollan durante el entrenamiento y la competición. Tales datos pueden ser muy útiles para los entrenadores, al mejorar sus recursos, algo que puede resultar decisivo para su éxito profesional (Abad-Robles et al., 2014) y aumentar, al mismo tiempo, la calidad de los programas de formación en etapas de aprendizaje en categorías de fútbol-base (Bales, 2006).

Para el registro y análisis de las tareas de entrenamiento en deporte base, se han creado diferentes herramientas objetivas que categorizan esas tareas, entre las que se encuentra el instrumento denominado Sistema Integral para el Análisis de las Tareas de Entrenamiento (en adelante, SIATE) (Ibáñez et al., 2016). 
Concretamente en fútbol-base, existen estudios que emplean el SIATE para conocer los medios de iniciación al entrenamiento (Gómez-Carmona et al., 2018), las tareas diseñadas por los entrenadores de fútbol-base (Gamonales et al., 2020), las diferencias entre equipos bajo la responsabilidad de entrenadores con diferentes experiencias y formación (Gamonales, Gómez-Carmona, León, et al., 2019), las distinciones en la carga objetiva en función de variables de carga externa subjetiva, así como la relación entre los métodos de cuantificación utilizados (Gómez-Carmona, Gamonales, Feu e Ibáñez, 2019), e incluso, el perfil autodefinido del entrenador que permite conocer su modo de actuar (Gamonales, Gómez-Carmona, Córdoba-Caro e Ibáñez, 2019; Urbano-Arévalo et al., 2020). Además, se han realizado indagaciones que analizan la enseñanza del fútbol en el ámbito educativo (García-Ceberino, Feu e Ibáñez, 2019), identificando las asociaciones en los aprendizajes, tras someter a los estudiantes a 2 programas de intervención basados en la instrucción directa y los juegos tácticos para la enseñanza del fútbol. En la misma línea, García-Ceberino et al. (2020) examinan y confrontan la relación, con la metodología de enseñanza y el género, de la carga externa e interna resultante de la aplicación de 2 programas diferentes. Y, por último, García-Ceberino, Gamero, Feu e Ibáñez (2019) investigan la incidencia que tienen los medios de iniciación empleados en las clases de educación física para la enseñanza del fútbol.

Por tanto, el examen de la bibliografía consultada muestra la necesidad de ampliar el conocimiento sobre las características de las tareas de entrenamiento en fútbol-base, en general, y en relación con el diseño de las tareas con portero, en particular. De esta manera, los objetivos del presente estudio se centraron en a) analizar las diferencias entre la variable presencia de portero $(P O R)$ y las variables pedagógicas que influyen en el diseño de tareas y en b) conocer el modo de actuación del entrenador de fútbol-base, categoría Benjamín (en adelante, categoría sub-10), que elaboró las sesiones de entrenamiento.

\section{MÉTodo}

\section{Diseño}

Este estudio se incluye en una estrategia descriptiva de tipo selectivo (Ato et al., 2013), con la finalidad de conocer la planificación y selección de tareas de entrenamiento de un entrenador en fútbol-base de categoría sub-10.

\section{Muestra}

En esta investigación, se ha analizado un total de 12 sesiones correspondientes a la etapa formativa en fútbol, desarrollada entre enero y febrero de 2020. Durante las 12 sesiones, se categorizó un total de 46 tareas, diseñadas por un único entrenador, perteneciente a un club de fútbol federado de categoría sub-10, que juega en una liga local de la Comunidad Autónoma de Extremadura (España), concretamente en la localidad de Cáceres. En el momento del estudio, el entrenador tenía una experiencia de 8 años y formación deportiva como técnico deportivo de fútbol de nivel III. Fue notificado de las características de la indagación, así como de los métodos y procedimientos asociados a su participación, firmando un consentimiento informado antes del inicio de la recogida de datos. En la tabla 1, se muestra el tiempo empleado en las diferentes sesiones planificadas. 
MHSALUD, ISSN: 1659-097X, 18(1), ENERo-Junio, 2021, PP 1-14

GAMONALES, León y MuÑoZ-JimÉnEZ

TABLA 1.

Análisis de la duración de las sesiones analizadas (en segundos)

\begin{tabular}{cccccc}
\hline Sesiones & Tareas & $\begin{array}{c}\text { Tiempo total } \\
\text { * }\end{array}$ & $\begin{array}{c}\text { Tiempo explicación } \\
{ }^{*}\end{array}$ & $\begin{array}{c}\text { Tiempo útil }^{*} \\
\mathbf{M} \pm \mathbf{D E}\end{array}$ & $\begin{array}{c}\text { Aprovechamiento }^{* *} \\
\mathbf{M} \pm \mathbf{D E}\end{array}$ \\
\hline Sesión 1 & 6 & $800.0 \pm 451.60$ & $65.0 \pm 29.40$ & $735.0 \pm 426.60$ & $\mathbf{M} \pm \mathbf{D E}$ \\
Sesión 2 & 5 & $1020.0 \pm 268.3$ & $66.0 \pm 13.41$ & $954.0 \pm 263.50$ & $93.1 \pm 2.07$ \\
Sesión 3 & 3 & $1333.3 \pm 288.6$ & $60.0 \pm 0.00$ & $1260.0 \pm 311.70$ & $94.0 \pm 3.46$ \\
Sesión 4 & 5 & $1020.0 \pm 268.3$ & $84.0 \pm 13.41$ & $936.0 \pm 265.20$ & $91.1 \pm 3.46$ \\
Sesión 5 & 4 & $1200.0 \pm 424.2$ & $60.0 \pm 0.00$ & $1140.0 \pm 424.20$ & $94.5 \pm 1.59$ \\
Sesión 6 & 3 & $1500.0 \pm 0.00$ & $60.0 \pm 0.00$ & $1440.0 \pm 0.00$ & $96.0 \pm 0.00$ \\
Sesión 7 & 2 & $2250.0 \pm 1484.9$ & $60.0 \pm 0.00$ & $2190.0 \pm 1484.90$ & $96.5 \pm 2.24$ \\
Sesión 8 & 4 & $1200.0 \pm 244.9$ & $60.0 \pm 0.00$ & $1140.0 \pm 244.90$ & $94.8 \pm 1.10$ \\
Sesión 9 & 3 & $1150.0 \pm 606.2$ & $60.0 \pm 0.00$ & $1080.0 \pm 623.53$ & $90.6 \pm 9.23$ \\
Sesión 10 & 4 & $1125.0 \pm 618.4$ & $60.0 \pm 0.00$ & $1065.0 \pm 618.46$ & $93.1 \pm 3.69$ \\
Sesión 11 & 4 & $1275.0 \pm 450.0$ & $60.0 \pm 0.00$ & $1215.0 \pm 450.0$ & $94.8 \pm 1.75$ \\
Sesión 12 & 3 & $1333.3 \pm 288.6$ & $60.0 \pm 0.00$ & $1260,0 \pm 311.76$ & $94.0 \pm 3.46$ \\
Total & 46 & $1180.1 \pm 500.1$ & $63.9 \pm 13.57$ & $1121.7 \pm 500.73$ & $93.2 \pm 3.82$ \\
\hline
\end{tabular}

El tiempo planificado variaba en función de la complejidad de las tareas y la adquisición de los conocimientos por parte de los jugadores. Por ello, la distribución temporal de las sesiones ofrece información relevante a los entrenadores sobre aspectos organizativos del grupo y datos sobre el compromiso motor de los deportistas durante las sesiones. Además, el estudio se desarrolló sobre la base de las disposiciones éticas de la Declaración de Helsinki (2013), aprobado por el Comité de Bioética de la Universidad de Extremadura (n. ${ }^{\circ}$ de registro 67/2017).

\section{Instrumento y variables analizadas}

Las tareas de entrenamiento se registraron mediante la herramienta SIATE (Ibáñez et al., 2016). En la tabla 2 , se muestran las variables pedagógicas analizadas en el estudio. 
Tabla 2. Variables pedagógicas analizadas en el estudio

\begin{tabular}{|c|c|c|c|}
\hline Tipo & Variables pedagógicas & Nomenclatura & Definición \\
\hline \multirow[t]{4}{*}{ Independiente } & Presencia de portero & POR & $\begin{array}{l}\text { Indica si existe portero durante la tarea o, por el con- } \\
\text { trario, si se prescinde del rol en las agrupaciones. }\end{array}$ \\
\hline & Situación de juego & SJ & $\begin{array}{l}\text { Agrupaciones de jugadores que los entrenadores diseñan } \\
\text { para cada una de las tareas. En ellas se incrementa la } \\
\text { complejidad desde la situación de } 1 \mathrm{vs} 0 \text { hasta } 8 \mathrm{vs} 8 \text {. }\end{array}$ \\
\hline & Fase de juego & FJ & $\begin{array}{l}\text { Está determinada por la posesión del balón y se } \\
\text { divide en rol definido durante la tarea (ataque o } \\
\text { defensa) o fase mixta (ataque-defensa). Si no existe } \\
\text { el aspecto competitivo, la fase de juego se clasifica } \\
\text { en calentamiento, preparación física, recuperación o } \\
\text { actividades de evaluación. }\end{array}$ \\
\hline & Tipo de contenido & CONT-G & $\begin{array}{l}\text { Se agrupa en contenidos individuales, grupales y de } \\
\text { equipo, tanto para el desarrollo en fase de ataque } \\
\text { como de defensa, así como para la conductas tácticas } \\
\text { y gestos técnicos. }\end{array}$ \\
\hline \multirow{3}{*}{ Dependientes } & $\begin{array}{l}\text { Medio de iniciación } \\
\text { deportiva }\end{array}$ & MIE & $\begin{array}{l}\text { Representalas actividades motrices deportivas que sirven } \\
\text { para el desarrollo de los contenidos técnico-tácticos, } \\
\text { desde la menor complejidad (ejercicio de aplicación } \\
\text { simple) hasta la mayor (deporte y competición). }\end{array}$ \\
\hline & $\begin{array}{l}\text { Línea de juego / grupo } \\
\text { de jugadores }\end{array}$ & LJ & $\begin{array}{l}\text { Define la tarea por puestos específicos (portero, central, } \\
\text { lateral, medio, extremo o delantero) y en equipo. }\end{array}$ \\
\hline & Nivel de oposición & NO & $\begin{array}{l}\text { Identifica el tipo de oponente durante el desarrollo de la } \\
\text { tarea. Se clasifica en sin oposición, obstáculos estáticos, } \\
\text { obstáculos dinámicos, oposición modulada y oposición. }\end{array}$ \\
\hline
\end{tabular}

\section{Procedimiento}

Para analizar las tareas diseñadas por un entrenador de fútbol-base de categoría sub-10, así como para conocer las diferencias entre la variable presencia de portero (POR) y las variables pedagógicas que influyen en el diseño de tareas, el procedimiento estuvo formado por varias fases (figura 1 ):

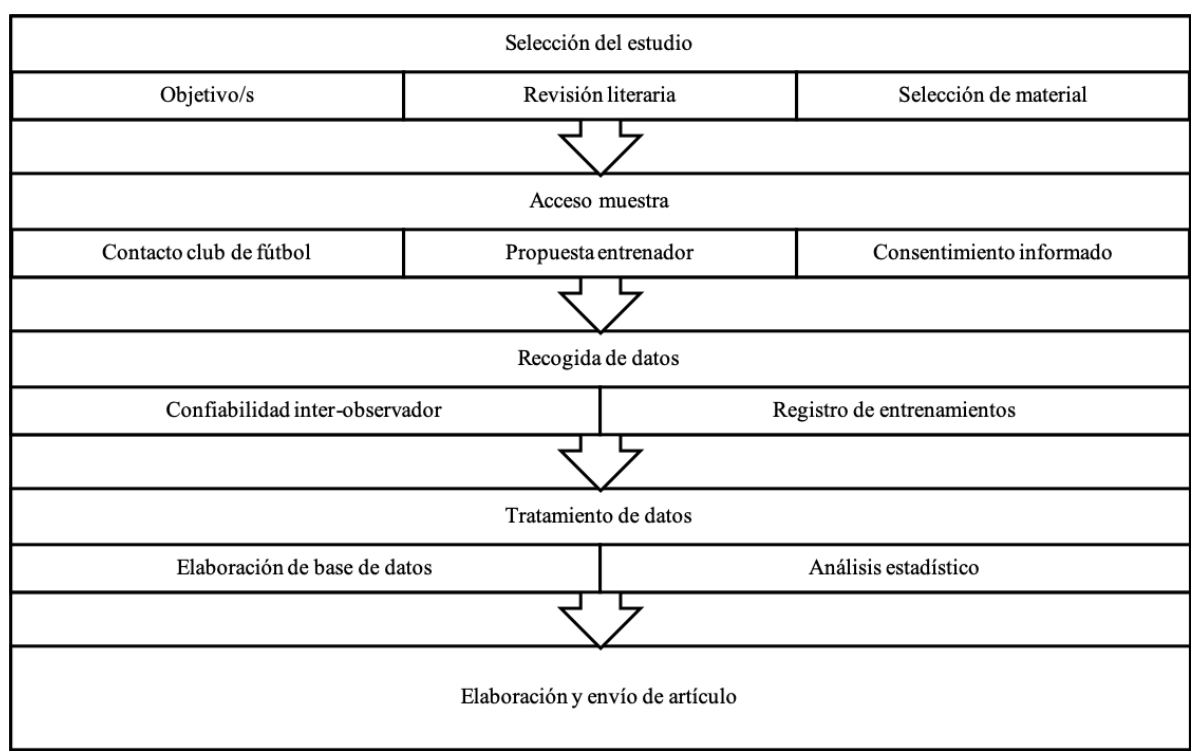

Figura 1. Fases del procedimiento del estudio 
Fase 1. Selección del estudio. Es un período relacionado con el tratamiento, la organización y la gestión de los datos. Para ello, se seleccionaron los objetivos por implementar, se desarrolló la búsqueda bibliográfica y se eligieron las herramientas por utilizar en la investigación.

Fase 2. Acceso muestra. En esta fase, los investigadores contactaron a la directiva del club de fútbol, con la finalidad de proponer el estudio. Una vez autorizado, el entrenador del equipo de fútbol-8 de categoría sub-10 participante en el trabajo firmó un consentimiento informado previo a la recogida de datos, tras explicarle las características de la investigación y su papel en esta.

Fase 3. Recogida de datos. Se utilizó el instrumento SIATE para el registro de las tareas de entrenamiento diseñadas por un único entrenador de fútbol de categoría sub-10, durante la etapa formativa, comprendida entre enero y febrero de 2020, así como para analizar las diferencias entre la variable presencia de portero $(P O R)$ y las variables pedagógicas que influyen en el diseño de tareas. En concreto, se usó el bloque de variables pedagógicas y variables organizativas. Para este proceso es necesario formar a un observador externo. El procedimiento de formación del codificador fue similar a los existentes en la literatura científica (Gamonales et al., 2020; Gamonales, Gómez-Carmona, León et al., 2019).

El codificador realizó un entrenamiento; se evaluó su fiabilidad mediante 2 registros de las mismas observaciones en diferentes sesiones, separadas convenientemente en el tiempo. La fiabilidad intercodificador se valoró mediante el índice Multirater Kappa Free (Randolph, 2005), que asume que un valor de 0.70 o superior indica un alto grado de acuerdo. El resultado fue como mínimo de 0.90 en todas las variables. Posteriormente, el observador registró todas las tareas de entrenamiento $(n=46)$, diseñadas por un único entrenador de fútbol-base, en la categoría sub-10, durante la etapa formativa.

Fase 4. Tratamiento de datos. Los datos fueron analizados mediante el software SPSS (versión 24, IBM, Armonk NY, EE. UU.). En esta fase, es importante la organización y categorización óptima de todas las variables utilizadas en el estudio, así como la ordenación del correcto filtrado de los datos.

Fase 5. Elaboración y envío de artículo. Una vez analizados los datos, los investigadores revisaron nuevamente la literatura relacionada con la investigación abordada, con el fin de seleccionar fuentes de utilidad. Posteriormente, redactaron cada uno de los apartados del artículo, escribiendo lo necesario para que la indagación se pueda reproducir en un futuro. Tras varias revisiones de los diferentes autores, se elaboró la versión final y se envió a la revista especializada.

\section{Análisis estadístico}

En primer lugar, se realizó un análisis descriptivo de todas las variables. Luego, para conocer las diferencias entre la variable presencia de portero $(P O R)$ y las variables pedagógicas que influyen en el diseño de tareas, se empleó chi cuadrado ( $\chi^{2}$ ) (Newell, Aitchison y Grant, 2014), identificando el nivel de asociación de las variables significativas mediante el coeficiente phi de Cramer ( $\phi$ c) y los valores definidos por Crewson (2006): (a) pequeño (<0.100), (b) bajo (0.100-0.299), moderado (0.300-0.499), (d) alto (>0.500).

Del mismo modo, se llevó a cabo la prueba exacta del test de Fisher (f), válido para todos los tamaños de la muestra, puesto que los resultados de la prueba de $\chi 2$ pueden ser inexactos cuando los conteos de celda son demasiado pequeños (De la Fuente-Fernández, 2016), como es el caso de esta investigación, en la cual existen conteos muy bajos en diversas categorías de las variables pedagógicas, como consecuencia del número de tareas analizadas. Por último, la interpretación del grado de asociación entre las variables se realizó mediante los residuos tipificados corregidos $(R T C)$ de las tablas de contingencia (Field, 2009) resultantes de cruzar las variables independientes del estudio (variables pedagógicas) con la variable dependiente $(P O R)$. 


\section{Resultados}

En la tabla 3, se muestran las diferencias entre la variable presencia de portero $(P O R)$ y las variables pedagógicas que influyen en el diseño de tareas de entrenamiento elaboradas por un entrenador de fútbolbase de categoría sub-10. Se han encontrado distinciones con un nivel de asociación alto, en las variables $S J$, FJ, CONT-G y MIE, y con uno moderado, la variable $N O$ (el resultado exacto, en lugar del resultado Monte Carlos, como consecuencia de la tabulación cruzada 2 × 2).

Tabla 3. Relación entre la variable presencia del portero (POR) y las variables independientes

\begin{tabular}{ccccccccc}
\hline \multirow{2}{*}{$\begin{array}{c}\text { Variables } \\
\text { pedagógicas }\end{array}$} & \multicolumn{7}{c}{ Presencia del portero } \\
\cline { 2 - 9 } & $\chi 2$ & $g l$. & $p$ & $\varphi \mathrm{c}$ & $p$ & $f$ & $p$ & $N A$ \\
\hline SJ & 29.860 & 80 & $\mathbf{0 . 0 0 2 *}$ & 0.805 & 0.002 & 30.856 & 0.000 & Alto \\
FJ & 21.369 & 3 & $\mathbf{0 . 0 0 0 *}$ & 0.682 & 0.000 & 21.399 & 0.000 & Alto \\
CONT-G & 25.474 & 8 & $\mathbf{0 . 0 0 1 *}$ & 0.744 & 0.001 & 25.981 & 0.000 & Alto \\
MIE & 21.206 & 5 & $\mathbf{0 . 0 0 1 *}$ & 0.679 & 0.001 & 21.786 & 0.000 & Alto \\
LJ & 5.193 & 2 & 0.075 & 0.336 & 0.075 & 4.895 & 0.052 & -- \\
NO & 9.036 & 1 & $\mathbf{0 . 0 0 3 *}$ & 0.443 & 0.003 & -- & -- & Moderado \\
\hline
\end{tabular}

${ }^{*} \mathrm{p}<0.05$ NA: Nivel de asociación según Crewson (2006)

Para la interpretación de las diferencias encontradas, se presentan los resultados de las variables significativas en la tabla 4, que incluye los residuos tipificados corregidos $(R T C)$, con la finalidad de determinar el grado de asociación entre las variables, las cuales puedan explicar el modo de actuación del entrenador en fútbol-base, en la categoría sub-10. 
MHSALUD, ISSN: 1659-097X, 18(1), ENERo-JunIo, 2021, PP 1-14

Gamonales, León y MuÑoZ-JimÉnez

Tabla 4. Resultados descriptivos y RTC de las variables pedagógicas

\begin{tabular}{|c|c|c|c|c|c|c|}
\hline \multirow{3}{*}{$\begin{array}{l}\text { Variables } \\
\text { Situación de Juego (SJ) }\end{array}$} & \multicolumn{5}{|c|}{ Presencia de portero } & \multirow[b]{3}{*}{ RTC } \\
\hline & \multicolumn{2}{|c|}{ Con portero } & \multicolumn{3}{|c|}{ Sin portero } & \\
\hline & $n$ & $\%$ & RTC & $n$ & $\%$ & \\
\hline $1 \times 0$ & 4 & 8.7 & -3.0 & 10 & 21.7 & 3.0 \\
\hline $1 \times 1$ & 1 & 2.2 & -0.3 & 1 & 2.2 & 0.3 \\
\hline $2 \times 1$ & 1 & 2.2 & 0.8 & 0 & 0.0 & -0.8 \\
\hline $2 \times 2$ & 1 & 2.2 & -0.3 & 1 & 2.2 & 0.3 \\
\hline $3 \times 1$ & 0 & 0.0 & -1.3 & 1 & 2.2 & 1.3 \\
\hline $3 \times 2$ & 0 & 0.0 & -1.3 & 1 & 2.2 & 1.3 \\
\hline $4 \times 0$ & 2 & 4.3 & 1.2 & 0 & 0.0 & -1.2 \\
\hline $4 \times 4$ & 2 & 4.3 & 1.2 & 0 & 0.0 & -1.2 \\
\hline $5 \times 2$ & 0 & 0.0 & -1.8 & 2 & 4.3 & 1.8 \\
\hline $5 \times 5$ & 3 & 6.5 & 1.4 & 0 & 0.0 & -1.4 \\
\hline $6 \times 2$ & 0 & 0.0 & -1.3 & 1 & 2.2 & 1.3 \\
\hline $8 \times 8$ & 16 & 34.8 & 4.0 & 0 & 0.0 & -4.0 \\
\hline Fase de juego (FJ) & $n$ & $\%$ & $R T C$ & $n$ & $\%$ & RTC \\
\hline Ataque & 6 & 13.0 & 2.1 & 0 & 0.0 & -2.1 \\
\hline Mixta & 22 & 47.8 & 2.4 & 8 & 17.4 & -2.4 \\
\hline Calentamiento & 0 & 0.0 & -4.2 & 9 & 19.6 & 4.2 \\
\hline Recuperación & 0 & 0.0 & -1.3 & 1 & 2.2 & 1.3 \\
\hline Tipo de contenido (CONT-G) & $n$ & $\%$ & $R T C$ & $n$ & $\%$ & RTC \\
\hline CTTIA & 3 & 6.5 & 1.4 & 0 & 0.0 & -1.4 \\
\hline GTTIA & 2 & 4.3 & 1.2 & 0 & 0.0 & -1.2 \\
\hline CTTGA & 4 & 8.7 & -1.1 & 5 & 10.9 & 1.1 \\
\hline GTTGA & 0 & 0.0 & -1.3 & 1 & 2.2 & 1.3 \\
\hline Pase & 0 & 0.0 & -1.8 & 2 & 4.3 & 1.8 \\
\hline Salidas & 0 & 0.0 & -1.3 & 1 & 2.2 & 1.3 \\
\hline Calentamiento & 4 & 8.7 & -2.3 & 8 & 17.4 & 2.3 \\
\hline Recuperación & 0 & 0.0 & -1.3 & 1 & 2.2 & 1.3 \\
\hline Partido entrenamiento & 15 & 32.6 & 3.8 & 0 & 0.0 & -3.8 \\
\hline Medio de iniciación entrenamiento & $n$ & $\%$ & RTC & $n$ & $\%$ & RTC \\
\hline Ejercicio de aplicación simple & 6 & 13.0 & -2.0 & 9 & 19.6 & 2.0 \\
\hline Ejercicio de aplicación compleja & 2 & 4.3 & -2.6 & 7 & 15.2 & 2.6 \\
\hline Juego simple inespecífico & 0 & 0.0 & -1.3 & 1 & 2.2 & 1.3 \\
\hline Predeporte / deporte adaptado & 3 & 6.5 & 0.6 & 1 & 2.2 & -0.6 \\
\hline Deporte & 5 & 10.9 & 1.9 & 0 & 0.0 & -1.9 \\
\hline Competición & 12 & 26.1 & 3.2 & 0 & 0.0 & -3.2 \\
\hline Línea de juego (LJ) & $n$ & $\%$ & RTC & $n$ & $\%$ & RTC \\
\hline Portero & 1 & 2.2 & -0.3 & 1 & 2.2 & 0.3 \\
\hline Central & 0 & 0.0 & -2.2 & 3 & 6.5 & 2.2 \\
\hline Equipo & 27 & 58.7 & 2.0 & 14 & 30.4 & -2.2 \\
\hline Nivel de oposición (NO) & $n$ & $\%$ & RTC & $n$ & $\%$ & RTC \\
\hline Sin oposición & 5 & 10.9 & -3.0 & 11 & 23.9 & 3.0 \\
\hline Con oposición & 23 & 50.0 & 3.0 & 7 & 15.2 & -3.0 \\
\hline
\end{tabular}

$\mathrm{RTC}=|1.96|$ 


\section{Discusión}

El estudio tiene como objetivo principal conocer si existe relación entre la variable presencia de portero (POR) y las variables pedagógicas que influyen en el diseño de tareas, ligamen que permita saber el modo de actuación de un entrenador de fútbol-base, de categoría sub-10, durante 12 sesiones de entrenamiento. Los resultados obtenidos muestran que el entrenador aprovecha, como mínimo, un $90 \%$ del tiempo de las sesiones. Además, el análisis descriptivo e inferencial expone que utiliza una reducida variedad de variables pedagógicas en sus entrenamientos, con el propósito de desarrollar y afianzar los aspectos básicos vinculados con el fútbolbase, en la categoría sub-10. Estos datos son diferentes a los obtenidos en estudios previos en fútbol de categoría juvenil (Gamonales, Gómez-Carmona, Córdoba-Caro et al., 2019; Gamonales, Gómez-Carmona, León et al., 2019), en los cuales los entrenadores emplean gran diversidad de variables pedagógicas en el diseño de las tareas. Esto evidencia que la herramienta SIATE proporciona cuantiosa cantidad de información enlazada a los factores que inciden en el entrenamiento de los deportes (Ibáñez et al., 2016), de manera fiable, independientemente de la modalidad y la categoría.

Los resultados presentan diferencias entre la variable $P O R$ y las variables pedagógicas que influyen en el diseño de tareas de entrenamiento elaboradas por un entrenador de fútbol-base, en la categoría sub-10; concretamente, en las variables pedagógicas SJ, FJ, CONT-G, MIE y NO. Sin embargo, la $L J$ no está influenciada por la $P O R$, lo que significa que no existen distinciones como consecuencia de diseñar tareas con más jugadores, en relación con el sistema o modelo de juego del equipo.

Respecto a la $S J$ y su asocie con la $P O R$, existe una mayor probabilidad de lo esperado de que el entrenador diseñe situaciones de juego $1 \times 0$ sin presencia de portero y también más allá de las expectativas de que las situaciones de entrenamiento de $8 \times 8$ sean con presencia de portero. Estudios previos dejan ver que no todos los entrenadores usan las mismas situaciones de juego para entrenar y, en función de la categoría, varían. En la categoría juvenil, se utiliza gran variedad de situaciones de juego (Gamonales, Gómez-Carmona, CórdobaCaro et al., 2019; Urbano-Arévalo et al., 2020) y suelen planificarse sin presencia de portero (Gamonales et al., 2020; Gamonales, Gómez-Carmona, León et al., 2019; Gómez-Carmona et al., 2018). Este hecho está acorde con la evolución del entrenamiento en fútbol de categorías inferiores, donde, en los últimos años, se está potenciando el trabajo por puesto específico. Asimismo, en la categoría cadete, se sugiere reforzar acciones defensivas como juego aéreo y estiradas; respecto a las acciones ofensivas, iniciar y continuar con la fase ofensiva, en coordinación con el equipo. Para ello, se debe trabajar, de forma específica, control, pase, saque a balón parado y saque con el balón en movimiento (Lapresa et al., 2018). Sería recomendable realizar investigaciones con mayor número de entrenadores, con el objetivo de conocer más a fondo la realidad de cómo se adecúan las tareas en función de la categoría.

Igualmente, se aprecian diferencias significativas entre las variables $P O R$ y $F J$. Existe una probabilidad mayor de lo esperado de que el entrenador diseñe tareas de entrenamiento mixtas y de ataque con presencia de portero, así como de calentamiento planeadas sin presencia de portero. Estos datos no corroboran lo resultados previos de la literatura científica, los cuales señalan que, dependiendo del perfil del entrenador (Gamonales, Gómez-Carmona, Córdoba-Caro et al., 2019; Urbano-Arévalo et al., 2020), el mes competitivo (Gamonales et al., 2020; Gamonales, Gómez-Carmona, León et al., 2019; Gómez-Carmona et al., 2018) y la categoría (Urbano-Arévalo et al., 2020), los entrenadores diseñan tareas de entrenamientos con diferentes FJ. Por tanto, es recomendable que los jugadores realicen entrenamientos específicos, tanto los de campo como los porteros, de forma independiente, para después trabajar de forma conjunta, con el fin de afianzar los conceptos básicos relacionados con cada posición de juego, ya que las demandas técnico-tácticas de los porteros son diferentes de los requerimientos del resto de jugadores (Gómez-Carmona et al., 2019). Además, respecto a los porteros, se aconseja diseñar tareas de entrenamiento que exijan acciones de alta intensidad (Di Salvo et al., 2008), en los niveles defensivo y ofensivo (Lapresa et al., 2018), al igual que trabajar aspectos psicológicos (Furley et al., 2016; Lidor et al., 2012; Montesano, 2016; Navarro et al., 2013). 
La variable $C O N T-G$ presenta diferencias respecto a la POR. El entrenador de fútbol-base, de categoría sub-10, diseña tareas con presencia de portero en los partidos de entrenamiento y manifiesta una menor probabilidad de lo esperado de que las tareas programadas de calentamiento sean sin presencia de portero. Los datos están en consonancia con la literatura científica previa, la cual comprueba que los entrenadores diseñan ejercicios de aplicación simple, como el $1 \times 0$, para el calentamiento o la preparación física (Gómez-Carmona et al., 2018), cuyo objetivo es desarrollar la condición física óptima para la realización del entrenamiento (Stølen et al., 2005). Los contenidos relacionados con el calentamiento se suelen implementar individualmente. Sin embargo, se recomienda llevar a cabo entrenamientos integrados, acorde con la tendencia actual del entrenamiento en fútbol-base (Shafizadeh et al., 2015), mediante juegos o activaciones en espacios reducidos con o sin balón.

Respecto a la $M I E$ y su nexo con la variable $P O R$, los resultados señalan diferencias significativas. Hay mayor probabilidad de lo esperado de que el entrenador diseñe ejercicios de aplicación compleja y ejercicios de aplicación simple sin la intervención del portero. Por el contrario, las tareas de entrenamiento relacionadas con la competición son planeadas con presencia de portero. Estos resultados son distintos a los presentes en la literatura científica previa en categoría juvenil (Gamonales, Gómez-Carmona, Córdoba-Caro et al., 2019; Gamonales, Gómez-Carmona, León et al., 2019; Gómez-Carmona et al., 2019), en los cuales predominan las tareas de predeporte, deporte adaptado y juegos en espacios reducidos. De igual modo, los datos indican que los porteros desempeñan un trabajo específico o son integrados en las tareas como jugadores de campo. Por ello, el diseño de las tareas de entrenamiento en fútbol-base debe partir de la realidad de la competición (Casamichana et al., 2013) y la idoneidad del espacio para desarrollar los entrenamientos (Urbano-Arévalo et al., 2020). Sería recomendable analizar varias temporadas, e incluso estudiar la progresión de los diferentes factores que influyen en las variables pedagógicas y el proceso formativo del deportista, así como conocer la influencia de que el equipo cuente con un segundo entrenador o ayudante durante los entrenamientos.

Hay diferencias significativas entre las variables $P O R$ y $N O$. En concreto, hay una probabilidad mayor de lo esperado de que las tareas de entrenamiento diseñadas con oposición por el entrenador, en la categoría sub-10, sean con presencia de portero, mientras que las de entrenamiento sin oposición son planificadas sin presencia de portero. Por tanto, en fútbol-base se deben proyectar tareas próximas al juego real (Urbano-Arévalo et al., 2020), con el fin de conseguir jugadores más completos (Gamonales, Gómez-Carmona, León et al., 2019). Para ello, los técnicos de fútbol-base deben dominar un amplio repertorio técnico de contenidos generales y específicos, así como mostrar una gran capacidad en la resolución de problemas (Urbano-Arévalo et al., 2020). En consecuencia, las tareas por diseñar y desarrollar tienen que estar en función de la edad y del nivel de exigencias de la competición (Gamonales, Gómez-Carmona, Córdoba-Caro et al., 2019).

Por último, no se muestran diferencias significativas entre las variables $P O R$ y $L J$. Sin embargo, el instructor diseñó tareas de entrenamiento de equipo con presencia de portero y, en menor medida, otras específicas relacionadas con las distintas posiciones de los jugadores en el terreno de juego (portero, lateral, central, mediocentro, extremo y delantero). Se halla muy poca literatura en torno a jóvenes futbolistas en las primeras etapas de formación y, concretamente, que analice la presencia del portero en las funciones relacionadas con la LJ. La bibliografía previa está centrada en la formación de los entrenadores de jóvenes futbolistas (AbadRobles et al., 2014). Por ende, resulta interesante profundizar en los diversos principios metodológicos empleados por los entrenadores de categorías de formación, con la idea de diseñar y desenvolver tareas de entrenamiento adecuadas a la edad de los futbolistas y su aplicabilidad al puesto específico del portero (Ortega-Toro y Sainz de Baranda, 2003). Para ello, se recomienda planificar tareas útiles para la formación integral de los jugadores, que tengan una transferencia positiva a la vida cotidiana. 


\section{Conclusiones}

El entrenador analizado en este estudio, durante 2 meses de la etapa formativa, en la categoría sub-10, diseña principalmente: tareas de $8 \times 8$, mixtas y competitivas con presencia de portero, mientras que las de entrenamiento sin presencia de portero más utilizadas son del tipo 1 x 0 y suelen estar orientadas a las actividades de calentamiento.

En relación con las diferencias entre la variable $P O R$ y las variables pedagógicas que influyen en el diseño de tareas de entrenamiento, las principales asociaciones se encuentran con SJ, FJ, CONT-G, MIE y NO. Por ello, es importante armar entrenamientos en los cuales se trabaje, de modo integral, las diferentes líneas de juego y con presencia de portero.

La herramienta SIATE utilizada en la presente investigación se muestra de nuevo útil y fiable para conocer el modo de actuación de cada entrenador de fútbol-base, así como para analizar los diferentes factores que inciden en el diseño de tareas de entrenamiento.

Teniendo en cuenta que en esta indagación se muestra un estudio de caso que recoge 2 meses competitivos de entrenamiento de un equipo federado de categoría sub-10, con un único entrenador analizado (que posee características propias de experiencia y formación), el trabajo es un excelente punto de partida, ya que ha demostrado una metodología correcta y eficaz de análisis de las tareas en fútbol-base. Por ello, para proyectos futuros, se debería profundizar en los objetivos de este, así como aumentar el número de entrenadores y tareas de entrenamiento, en las categorías iniciales de formación deportiva, etapas poco estudiadas.

\section{Financiación}

Este trabajo ha sido parcialmente subvencionado por la Ayuda a los Grupos de Investigación (GR18170) de la Junta de Extremadura (Consejería de Empleo e Infraestructuras); con la aportación de la Unión Europea, a través de los Fondos Europeos de Desarrollo Regional (FEDER).

\section{Conflicto de intereses}

Estudio desarrollado en el Grupo de Optimización del Entrenamiento y Rendimiento Deportivo (GOERD) de la Facultad de Ciencias del Deporte de la Universidad de Extremadura. Todos los autores hemos contribuido en la realización del manuscrito.

\section{REFERENCIAS}

Abad-Robles, M. T., Giménez, F. J., Robles, J. y Castillo, V. (2014). La formación de los entrenadores de jóvenes futbolistas. E-balonmano.com: Revista de Ciencias del Deporte, 9(2), 105-114. http://www.e-balonmano.com/o js/index.php/revista/article/view/116

Ato, M., López, J. J. y Benavente, A. (2013). Un sistema de clasificación de los diseños de investigación en psicología. Anales de Psicología, 29(3), 1038-1059. http://scielo.isciii.es/scielo.php?script=sci_arttext\&pid=S0212-97282 013000300043

Bales, J. (2006). Introduction: Coach education. The Sport Psychologist, 20, 126-127.

Bazmara, M., Jafari, S. y Pasand, F. (2013). A Fuzzy expert system for goalkeeper quality recognition. International Journal of Computer Science, 9(5), 1-5.

Casamichana, D., Castellano, J., Calleja-González, J., San Román, J. y Castagna, C. (2013). Relationship between indicators of training load in soccer players. The Journal of Strength \& Conditioning Research, 27(2), 369-374. $\mathrm{h}$ ttp://doi.org/10.1519/JSC.0b013e3182548af1 
Crewson, P. (2006). Applied statistics handbook. Version 1.2. Leesburg: AcaStat Software.

De la Fuente-Fernández, S. (2016). Aplicaciones de la Chi-cuadrado: Tablas de contigencias. Homogeneidad. Dependencia e independencia. Madrid: Universidad Auto\#noma de Madrid.

Di Salvo, V., Benito, P. J., Calderon, F. J., Di Salvo, M. y Pigozzi, F. (2008). Activity profile of elite goalkeepers during football matchplay. Journal of Sports Medicine and Physical Fitness, 48(4), 443-446. PMID: 18997646

Dicks, M. Uehara, L. y Lima, C. (2011). Deception, Individual Differences and Penalty Kicks: Implications for Goalkeeping in Association Football. International Journal of Sports Science e Coaching, 6(4), 515-521. https: //doi.org/10.1260/1747-9541.6.4.515

Field, A. (2009). Discovering statistics using SPSS (3.. ed.). London: Sage Publications Ltd.

Furley, P., Noël, B. y Memmert, D. (2016). Attention towards the goalkeeper and distraction during penalty shootouts in association football: a retrospective analysis of penalty shootouts from 1984 to 2012. Journal of Sports Sciences, 35(9), 873-879. https://doi.org/10.1080/02640414.2016.1195912

Gamonales, J. M., Gómez-Carmona, C. D., Córdoba-Caro, L. G. e Ibán\#ez, S. J. (2019). Influencia del perfil de entrenador en el disen\#o de las tareas en el fu\#tbol. Estudio de caso. Journal of Sport and Health Research, 11(1), 69-82.

Gamonales, J. M., Gómez-Carmona, C. D., León, K., García-Santos, D., Gamero-Portillo, M. G. y Muñoz-Jiménez, J. (2019). Análisis de las tareas de Entrenamiento en fútbol-base: diferencias entre dos meses durante el periodo competitive en la categoría sub-19. Sportis: Revista Técnico-Cientifica del Deporte Escolar, Educación Física y Psicomotricidad, 5(1), 30-52. http://doi.org/10.17979/sportis.2019.5.1.3469

Gamonales, J. M., Gómez-Carmona, C. D., León, K., Muñoz-Jiménez, J. e Ibáñez, S. J. (2020). Estudio de las variables pedagógicas en tareas de entrenamiento en fútbol-base según el mesociclo competitivo. Un estudio de casos. RETOS: Nuevas Tendencias en Educación Física, Deporte y Recreación, 37(1), 486-492. https://doi.org/10.471 97/retos.v37i37.71401

García-Ceberino, J. M., Antúnez, A., Feu, S. e Ibáñez, S. J. (2020). Quantification of Internal and External Load in School Football According to Gender and Teaching Methodology. International Journal of Environmental Research and Public Health, 17(1), 344. http://doi.org/10.3390/ijerph17010344.

García-Ceberino, J. M., Gamero, M. G., Feu, S. e Ibáñez, S. J. (2019). The mean of teaching as a determinant of the external Training Load of the tasks used to teach school soccer. ESHPA: Education, Sport, Health and Physical Activity, 3(3), 412-427. http://doi.org/10481/48260.

García-Ceberino, J. M., Feu, S. e Ibáñez, S. J. (2019). Comparative study of two intervention programmers for teaching soccer to school-age students. Sports, 7(3), 1-16. http://doi.org/10.3390/sports7030074

Gegenfurtner, A., Lehtinen, E. y Saljo, R. (2011). Expertise Differences in the Comprehension of Visualizations: A Meta-Analysis of Eye-Tracking Research in Professional Domains. Educational Psychology Review, 23(4), 523-552. https://doi.org/10.1007/s10648-011-9174-7

Gómez-Carmona, C. D., Gamonales, J. M., Feu, S. e Ibáñez, S. J. (2019). Estudio de la carga interna y externa a través de diferentes instrumentos. Un estudio de casos en fútbol formativo. Sportis: Revista Técnico-Cientifica del Deporte Escolar, Educación Fisica y Psicomotricidad, 5(3), 444-468. http://doi.org/10.17979/sportis.2019.5.3.5464

Gómez-Carmona, C. D., García-Rubio, J., Muñoz-Jiménez, J. y Gamonales, J. M. (2018). Relación entre el medio de iniciación al entrenamiento y las variables pedagógicas que definen las tareas en el fútbol. TRANCES: Revista de Transmisión del Conocimiento Educativo y de la Salud, 10(1), 401-420.

Gracia, F., García-Rubio, J., Cañadas, M. e Ibáñez, S. J. (2014). Diferencias en la frecuencia cardíaca en situaciones de juego modificadas en baloncesto de formación. E-balonmano.com: Revista de Ciencias del Deporte, 10(1), 23-30. http://www.e-balonmano.com/ojs/index.php/revista/article/view/137

Hägglund, M., Waldén, M. y Ekstrand, J. (2013). Risk factors for lower extremity muscle injury in professional soccer the UEFA injury study. The American Journal of Sports Medicine, 41(2), 327-335. https://doi.org/https://doi. org/10.1177/0363546512470634 
Ibáñez, S. J., Feu, S. y Cañadas, M. (2016). Sistema integral para el análisis de las tareas de entrenamiento, SIATE, en deportes de invasión. E-balonmano.com: Revista de Ciencias del Deporte, 12(1), 3-30. http://www.e-balonmano .com/ojs/index.php/revista/article/view/331/0

Lapresa, D., Chiviti-Navacués, J., Arana-Idiakez, J., Anguera, M.T. y Barbero-Cadirat, J. M. (2018). Análisis de la eficacia del portero cadete (14 a 16 años). Apunts: Educación Física y Deportes, 131(1), 60-79. https://doi.org/ 10.5672/apunts.2014-0983.es.(2018/1).131.05

Lidor, R., Ziv, G. y Gershon, T. (2012). Psychological Preparation of Goalkeepers for the 11-m Penalty Kick in SoccerA Review. Sport Psychologist, 26(3), 375-389. https://doi.org/10.1123/tsp.26.3.375

Mancha-Triguero, D., García-Ceberino, J. M., Antúnez, A. y García-Rubio, J. (2018). ¿Afecta la fase de juego al diseño de las tareas de un equipo de baloncesto de formación? SPORT-TK: Revista Euroamericana de Ciencias del Deporte, 7(2), 27-36. https://doi.org/10.6018/sportk.343201

Montesano, P. (2016). Goalkeeper in soccer: performance and explosive strength. Journal of Physical Education and Sport, 16(1), 230-233. https://doi.org/10.7752/jpes.2016.01036

Navarro, M., Van der Kamp, J., Ranvaud, R. y Savelsbergh, G. J. (2013). The mere presence of a goalkeeper affects the accuracy of penalty kicks. Journal of Sports Sciences, 31(9), 921-929. https://doi.org/10.10 80/02640414.2012.762602

Newell, J., Aitchison, T. y Grant, S. (2014). Statistics for sports and exercise science: a practical approach. London and New York: Routledge Taylor \& Francis Group.

Oberstone, J. (2010). Comparing English Premier League Goalkeepers: identifying the pitch actions that differentiate the best from the rest. Journal of Quantitative Analysis in Sports, 6(1), 1-9. https://doi.org/10.2202/1559-041 0.1221

Ortega-Toro, E. y Sainz de Baranda, P. (2003). El diseño de tareas en el fútbol-base: su aplicabilidad al puesto específico del portero. Cuadernos de Psicología del Deporte, 3(1), 15-32. https://revistas.um.es/cpd/article/view/112281

Pidemunt, G., Torres-Claramunt, R., Gines, A., de Zabala, S. y Cebamanos, J. (2012). Bilateral Stress Fracture of the Carpal Scaphoid: Report in a Child and Review of the Literature. Clinical Journal of Sport Medicine, 22(6), 511-513. https://doi.org/10.1097/JSM.0b013e3182580e03

Putukian, M. (2004). Heading in soccer: is it safe? Current Sports Medicine Reports, 3(1), 9-14. https://doi.org/10.1 007/s11932-004-0039-4

Randolph, J. J. (2005). Free-Marginal Multirater Kappa (Multirater Kfree): An Alternative to Fleiss i Fixed-Marginal Multirater Kappa. Joensuu Learning and Instruction Symposium 2005, University of the Joensuu, Finland.

Sáenz-López, P., Feu, S. e Ibáñez, S. J. (2006). Estudio de la participación de los jugadores españoles de baloncesto en las distintas categorías de la selección nacional. Apunts: Educación Física y Deporte, 85, 36-45.

Sainz de Baranda, P., Ortega-Toro, E. y Palao, J. M. (2008). Study of goalkeeper's defense in the World Cup in Korea and Japan in 2002. European Journal of Sport Science, 8(3), 127-134. https://doi.org/10.1080/174613908019 19045

Sainz de Baranda, P., Ortega-Toro, E., Llopis-Portugal, L., Novo-Palacios, J. F. y Rodríguez-Muñoz, D. (2005). Analysis of the goal keeper's defensive actions in soccer 7. Apunts: Educación Física y Deportes, 80, 45-52.

Shafizadeh, M., Davids, K., Correia, V., Wheat, J.y Hizan, H. (2015). Informational constraints on interceptive actions of elite football goalkeepers in 1v1 dyads during competitive performance. Journal of Sports Sciences 34(17) 1596-1601. https://doi.org/10.1080/02640414.2015.1125011

Silva, A., Sánchez-Bañuelos, F., Garganta, J. y Anguera, M. T. (2005). Patrones de juego en el fútbol de alto rendimiento. Análisis secuencial del proceso ofensivo en el campeonato del mundo Corea-Japón 2002. Cultura, Ciencia y Deporte, 1, 65-72.

Stølen, T., Chamari, K., Castagna, C. y Wisløff, U. (2005). Physiology of soccer. Sports Medicine, 35(6), 501-536.

Urbano-Arevalo, F. J., Mancha-Triguero, D., Gómez-Carmona, C. D. y Gamonales, J. M. (2020). Influencia del perfil del entrenador en el diseño de tareas en fútbol-base. Estudio de casos. RETOS: Nuevas Tendencias en Educación Física, Deporte y Recreación, 38(2), 204-212.

\section{BY-NC-ND}

